\title{
A POTENTIAL WELL THEORY FOR THE WAVE EQUATION WITH NONLINEAR SOURCE AND BOUNDARY DAMPING TERMS
}

\author{
ENZO VITILLARO \\ Dipartimento di Matematica ed Informatica, Universita di Perugia, \\ Via Vanvitelli,1 06123 Perugia, Italy \\ email; enzo@unipg.it
}

(Received 17 November, 2000; accepted 9 March 2001)

\begin{abstract}
The paper deals with local existence, blow-up and global existence for the solutions of a wave equation with an internal nonlinear source and a nonlinear boundary damping. The typical problem studied is

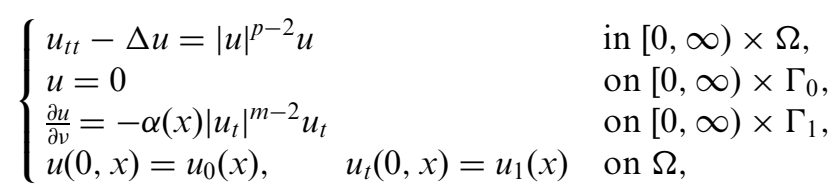

where $\Omega \subset \mathbb{R}^{n}(n \geq 1)$ is a regular and bounded domain, $\partial \Omega=\Gamma_{0} \cup \Gamma_{1}, \lambda_{n-1}\left(\Gamma_{0}\right)>0$, $2<p \leq 2(n-1) /(n-2)$ (when $n \geq 3), m>1, \alpha \in L^{\infty}\left(\Gamma_{1}\right), \alpha \geq 0$, and the initial data are in the energy space. The results proved extend the potential well theory, which is well known when the nonlinear damping acts in the interior of $\Omega$, to this problem.
\end{abstract}

2000 Mathematics Subject Classification. 35L70 (35L05, 35D05).

1. Introduction. We study the problem

$$
\begin{cases}u_{t t}-\Delta u=f(x, u) & \text { in }[0, \infty) \times \Omega, \\ u=0 & \text { on }[0, \infty) \times \Gamma_{0}, \\ \frac{\partial u}{\partial v}=-Q\left(x, u_{t}\right) & \text { on }[0, \infty) \times \Gamma_{1}, \\ u(0, x)=u_{0}(x), u_{t}(0, x)=u_{1}(x) & \text { on } \Omega,\end{cases}
$$

where $u=u(t, x), t \geq 0, x \in \Omega, \Delta$ denotes the Laplacian operator, with respect to the variable $x, \Omega$ is a regular and bounded domain of $\mathbb{R}^{n}(n \geq 1), \partial \Omega=\Gamma_{0} \cup \Gamma_{1}$, $\Gamma_{0} \cap \Gamma_{1}=\emptyset$, and $\lambda_{n-1}\left(\Gamma_{0}\right)>0\left(\lambda_{n-1}\right.$ denoting the $(n-1)$-dimensional Lebesgue measure on $\partial \Omega$ ). The initial data are in the energy space, that is $u_{0} \in H^{1}(\Omega)$ and $u_{1} \in L^{2}(\Omega)$, with the compatibility condition $u_{0}=0$ on $\Gamma_{0}$. Moreover $Q$ represents a nonlinear boundary damping, i.e. $Q(x, v) v \geq 0$, and $f$ is an internal nonlinear source, i.e. $f(x, u) u \geq 0$.

Work done in the framework of the M.U.R.S.T. project "Metodi variazionali ed equazioni differenziali nonlineari". 
There is a wide literature on problem (1), when $f(x, u) u \leq 0$ (and $Q(x, v) v \geq 0$ ). In this case, under various assumptions on $Q$ and $f$, global existence and decay estimates were proved for arbitrarily large initial data. See for example [5], [6], [7], [8], [16], [17], [18], [20] and [38].

Much less is known when $f$ is a source term. The only paper in this setting, in the author's knowledge, is [19], where global existence and exponential decay for small initial data is proved for nonlinearly perturbed wave equations.

In the particular case $\Gamma_{1}=\emptyset$ (the same arguments work also when $\left.Q \equiv 0\right)$, when $f(x, u)=|u|^{p-2} u, p>2$, it is well known that the source term causes blow-up of solutions in finite time for sufficiently large initial data. See for example [2], [12], [13], [14], [15], [21], [22], [29], [34]. We also refer to the related papers [23] and [24], dealing with boundary source terms.

In [29] some sharper results, known as a "potential well theory", were proved when $2<p<2^{*}$, where as usual $2^{*}$ is the Sobolev critical exponent $2 n /(n-2)$ when $n \geq 3,2^{*}=\infty$ when $n=1,2$. To illustrate this theory briefly let us introduce some notations: $\|\cdot\|_{p}=\|\cdot\|_{L^{p}(\Omega)}$, the functional

$$
J(u)=\frac{1}{2}\|\nabla u\|_{2}^{2}-\frac{1}{p}\|u\|_{p}^{p}, \quad u \in H_{0}^{1}(\Omega)
$$

the energy function

$$
E(t)=\frac{1}{2}\left\|u_{t}(t)\right\|_{2}^{2}+J(u(t))
$$

associated to a solution $u$ of (1), and the (positive) number

$$
d=\inf _{u \in H_{0}^{1}(\Omega), u \neq 0} \sup _{\lambda<0} J(\lambda u) .
$$

Moreover the value $d$ is shown to be the Mountain Pass level associated to the underlying Dirichlet problem $-\Delta u=|u|^{p-2} u$ in $\Omega, u=0$ on $\partial \Omega$.

In [29] it is proved that, if $E(0)<d$, the solution is global when $\nabla J\left(u_{0}\right) u_{0}>0$, while $^{1}$ it blows-up in finite time when $\nabla J\left(u_{0}\right) u_{0}<0$ (note the case $\nabla J\left(u_{0}\right) u_{0}=0$ is impossible since $E(0)<d$ ). One can easily see (the simple proof can be founded in [36]) that

$$
\begin{aligned}
& E(u(0))<d, \quad \nabla J\left(u_{0}\right) u_{0}<0 \quad \Longleftrightarrow \quad\left(\left\|\nabla u_{0}\right\|_{2}, E(0) \in A,\right. \\
& E(u(0))<d, \quad \nabla J\left(u_{0}\right) u_{0}<0 \quad \Longleftrightarrow \quad\left(\left\|\nabla u_{0}\right\|_{2}, E(0) \in B,\right.
\end{aligned}
$$

where $A$ and $B$ are defined (see Figure 1) by

$$
\begin{aligned}
& A=\left\{(\lambda, E) \in[0, \infty) \times \mathbb{R}: g(\lambda) \leq E<d, \quad \lambda<\lambda_{1}\right\}, \\
& \left.B=\{(\lambda, E)\} \in[0, \infty) \times \mathbb{R}: g(\lambda) \leq E<d, \quad \lambda>\lambda_{1}\right\},
\end{aligned}
$$

where $g(\lambda)=\frac{1}{2} \lambda^{2}-B_{1}^{p} \lambda^{p} / p, \lambda>0, B_{1}$ is the optimal constant of the Sobolev embedding, $\lambda_{1}$ is the absolute maximum point of $g$, and finally $d=g\left(\lambda_{1}\right)>0$.

${ }^{1}$ here $\nabla J$ denotes the gradient vector associated to the Fréchet differential of $J$ on $H_{0}^{1}(\Omega)$, that is $\nabla J\left(u_{0}\right) u_{0}=\left\|\nabla u_{0}\right\|_{2}^{2}-\left\|u_{0}\right\|_{p}^{p}$. 


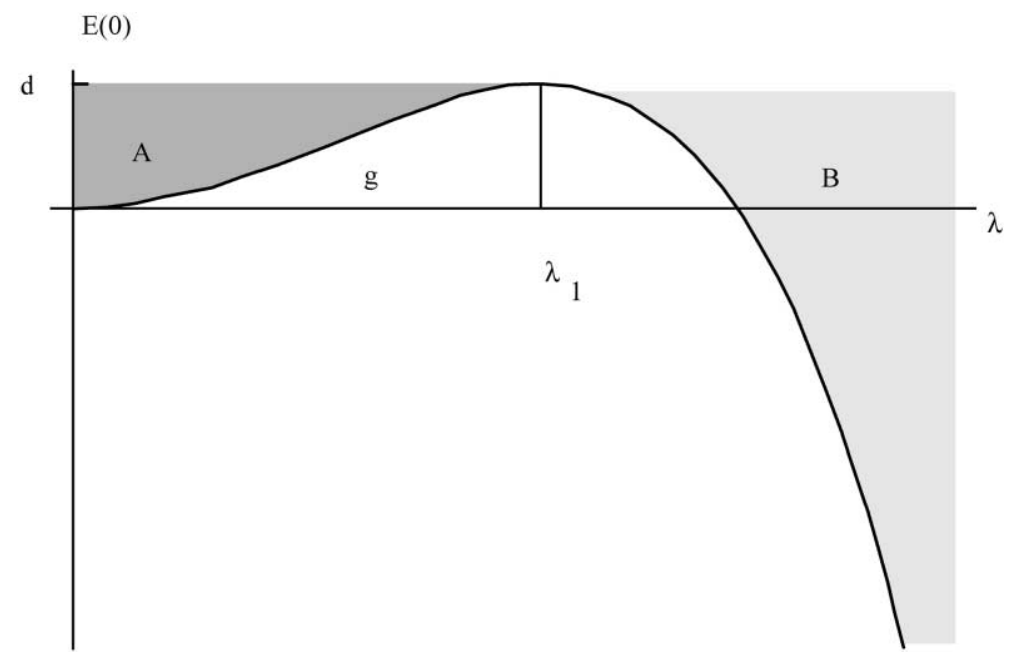

Figure 1. The sets $A$ and $B$ in the plane $(\lambda, E(0))$, where $\lambda=\left\|\nabla u_{0}\right\|_{2}$.

Blow-up for large initial data was proved in [11] and [25] when the dissipation arises from an internal nonlinear damping term, that is for solutions of the problem

$$
\begin{cases}u_{t t}-\Delta u+\left|u_{t}\right|^{m-2} u_{t}=|u|^{p-2} u & \text { in }[0, \infty) \times \Omega \\ u=0 & \text { on }[0, \infty) \times \partial \Omega \\ u(0, x)=u_{o}(x), \quad u_{t}(0, x)=u_{1}(x) & \text { on } \Omega\end{cases}
$$

when $2<p \leq 1+2^{*} / 2$ and $m<p$ (in the case $p \leq \min \left\{m, 1+2^{*} / 2\right\}$ global existence for arbitrary large initial data is proved in [11]). The extension to (5) of the potential well theory illustrated above was established in [30] when $m=2$, and in [35] when $m<1$.

These results known for the case $\Gamma_{1}=\emptyset$ motivate us to study (1) when, roughly, $f(x, u) \simeq|u|^{p-2} u, p>2$ or $f(x, u) \simeq|u|^{p-2} u-|u|^{q-2} u, 1<q<p, p>2$, where the second nonlinear term plays the role of a nonlinear perturbation acting against blowup. The case $f(x, u)=|u|^{p-2} u, p>2$ and $Q(x, v)=\alpha(x) v, \alpha \in L^{\infty}\left(\Gamma_{1}\right)$, was studied in [36], where the global nonexistence part of the potential well theory was established. The classical concavity argument used there, however, is no longer applicable when the damping $Q$ is nonlinear. Indeed, also local existence of the solutions of (1) must be proved when $Q$ is nonlinear, since classical nonlinear semigroup theory cannot be applied directly in this situation (see [28]).

The assumptions on $Q$ allow, for instance, to consider homogenous damping terms of the type $Q(x, v)=\alpha(x)|v|^{m-2} v, m>1, \alpha \in L^{\infty}\left(\Gamma_{1}\right), \alpha \geq 0$. The motivation to consider $\alpha(x) \not \equiv 1$, and in particular $\inf _{\Gamma_{1}} a=0$, comes from the case $f \equiv 0$ (or $f(x, u) u \leq 0)$, studied in [5], [6], [7], [8], [16], [17], [18], [20] and [38]. Moreover, in these papers, a damping term of the form $Q(x, \mathrm{v})=\left(\mathrm{x}-\mathrm{x}_{0}\right) v(\mathrm{x}) \mathrm{g}(\mathrm{v})$, where $x_{0}$ is a fixed point of $\mathbb{R}^{n}, v$ is the outward normal vector field on $\partial \Omega$ and the partition $\partial \Omega=\Gamma_{0} \cup \Gamma_{1}$ is given by

$$
\Gamma_{0}=\left\{x \in \partial \Omega:\left(x-x_{0}\right) \nu(x) \leq 0\right\}, \quad \Gamma_{1}=\left\{x \in \partial \Omega:\left(x-x_{0}\right) \nu(x)>0\right\}
$$


was considered; and exponential (when $g$ is linear near 0), or algebraic (when $g$ is superlinear near 0 ) decay was also proved as $t \rightarrow \infty$. When $\Omega$ is a simply connected region $\inf _{\Gamma_{1}}\left(x-x_{0}\right) v(x)=0$. This shows the interest, in studying blow-up phenomenam for damping terms of this type, since they produce boundary stabilization in absence of source.

Our study also includes the case $Q(x, v)=\alpha(x)\left(|v|^{m-2} v+v\right), \alpha \in L^{\infty}\left(\Gamma_{1}\right), m>1$, which describes a more realistic dissipation rate, linear for small $v$ and superlinear for large $v$ (see for example [9]). Roughly, to include both cases, we shall consider $Q(x, v) \simeq \alpha(x)\left(|v|^{\mu-2} v+|v|^{\mathrm{m}-2} v\right), 1<\mu \leq \mathrm{m}$.

To illustrate in a simple way our results we consider the case $f(x, u)=|u|^{p-2} u$ and $u=2$, i.e.

$$
\begin{cases}u_{t t}-\Delta u=|u|^{p-2} u & \text { in }[0, \infty) \times \Omega \\ u=0 & \text { on }[0, \infty) \times \Gamma_{0} \\ \frac{\partial u}{\partial v}=-\alpha(x)\left(\left|u_{t}\right|^{m-2} u_{t}+\beta u_{t}\right) & \text { on }[0, \infty) \times \Gamma_{1} \\ u(0, x)=u_{0}(x), \quad u_{t}(0, x)=u_{1}(x) & \text { on } \Omega\end{cases}
$$

where $m>1, \beta \geq 0, \alpha$ is a measurable nonnegative function on $\Gamma_{1}$. Again to a solution $u$ of (6) we associate the energy function given by (3). We denote by $L^{m}\left(\Gamma_{1}, \alpha\right)$ the $L^{m}$ space on $\Gamma_{1}$ associated to the measure $\mu_{\alpha}$ defined by $\mu_{\alpha}(A)=\int_{A} \alpha(x) d x$ for any measurable subset $A$ of $\Gamma_{1}$, and $L^{m}\left(\Gamma_{1}, 1\right)$ denotes the standard $L^{m}$ space associated to $\lambda_{n-1}$, that is $L^{m}\left(\Gamma_{1}\right)=L^{m}\left(\Gamma_{1}, 1\right)$. The analogous convention will be adopted on $(0, T) \times \Gamma_{1}$, for $T>0$. Moreover

$$
H_{\Gamma_{0}}^{1}(\Omega)=\left\{u \in H^{1}(\Omega):\left.u\right|_{\Gamma_{0}}=0\right\}
$$

(where $\left.u\right|_{\Gamma_{0}}$ is intended in trace sense), equipped with the norm $\|\nabla u\|_{2}$, which is equivalent, by the Poincaré inequality (see [37]), to the standard one since $\lambda_{n-1}\left(\Gamma_{0}\right)>0$.

Our first result concerns local existence for solutions of (6).

Theorem 1. Suppose that $2<p \leq 1+2^{*} / 2$, and that $u_{0} \in H_{\Gamma_{0}}^{1}(\Omega), u_{1} \in L^{2}(\Omega)$. Then there is $T>0$ and a unique weak solution $u$ of $(6)$ in $(0, T) \times \Omega$, such that

$$
\begin{gathered}
u \in C\left([0, T] ; H_{\Gamma_{0}}^{1}(\Omega)\right) \cap C^{1}\left([0, T] ; L^{2}(\Omega)\right), \\
u_{t} \in L^{m}\left((0, T) \times \Gamma_{1}, \alpha\right), \\
u_{t} \in L^{2}\left((0, T) \times \Gamma_{1}, \alpha\right), \quad \text { if } \beta>0,
\end{gathered}
$$

and $u$ satisfies the energy identity

for $0 \leq s \leq t$

$$
E(t)-E(s)=-\int_{s}^{t} \int_{\Gamma_{1}} \alpha(x)\left(\left|u_{t}\right|^{m}+\beta\left|u_{t}\right|^{2}\right)
$$

REMARK 1 . The regularity given by (8) and (eventually) (9) is not a consequence of the trace theorem, but is a further regularity property of $u$ insured by the presence of the damping term. 
The global existence theorem for initial data $u_{0}, u_{1}$ such that the corresponding couple $\left(\left\|\nabla u_{0}\right\|_{2}, E(0)\right)$ is in the region $A$.

Theorem 2. If the assumptions of Theorem 1 hold and $\left(\left\|\nabla u_{0}\right\|_{2}, E(0)\right) \in A$ then $u$ can be extended to $[0, \infty)$, and $\left(\|\nabla u(t)\|_{2}, E(t)\right) \in A$ for all $t \geq 0$.

REMARK 2. Theorem 1 characterizes in a variational way the neighborhood of stability of the null solution in which global existence holds, as shown in [19].

Our final result concerning (6) extends to the case $\Gamma_{1} \neq \emptyset$ the blow-up theorem of [29].

Theorem 3. Assume that the hypotheses of Theorem 1 hold, that $\alpha \in L^{\infty}\left(\Gamma_{1}\right)$ and $\left(\left\|\nabla u_{0}\right\|_{2}, E(0)\right) \in B$. Suppose moreover that

$$
m<m_{0}(p)=\frac{2(n+1) p-4(n-1)}{n(p-2)+4} .
$$

Then there is $T_{\max }<0$ such that $u$ blows-up in the $L^{p}$ norm of $\Omega$ as $t \rightarrow T_{\max }^{-}$.

REMARK 3. Assumption $\lambda_{n-1}\left(\Gamma_{0}\right)>0$, together with the Poincare inequality, yields that $\|\nabla u(t)\|_{2} \rightarrow \infty$ as $t \rightarrow T_{\text {max }}^{-}$, and of course the Hölder inequality implies also the blow-up in the $L^{\infty}$ norm of $\Omega$.

Assumption (11) strongly reduces the applicability of Theorem 3, see Figure 2, which illustrate the set of the couples $(p, m)$ satisfying the assumptions of Theorem 3. As $m_{0}(p)>2$ for $p>2$, the result is rather sharp in the sublinear case $1<m \leq 2$, while (11) and $p \leq 1+2^{*} / 2$ force that $m<4$ when $n=1, m<3$ when $n=2$ and $m<2+2 /(3 n-4)$ when $n \geq 3$. This is due to the difficulty in comparing the effect of high order dissipation, which is related to the $L^{m}$ norm on $\Gamma_{1}$, with the effect of the source, related to the $L^{p}$ norm on $\Omega$.

$\mathrm{m}$

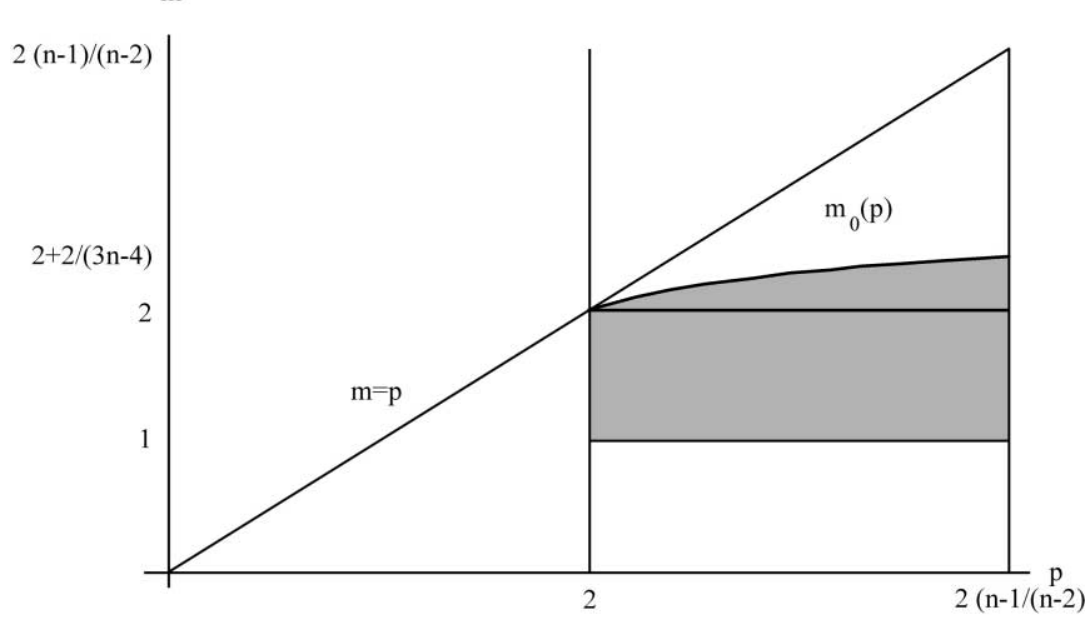

Figure 2. The shaded region is the set of the $(p, m)$ couples for which the assumptions of Theorem 3 hold, when $n \geq 3$. The picture is made in the case $n=3$. 
In this paper we shall consider nonlinearities $f$ and $Q$ more general than those in (6), as we indicated above. In particular also the problem

$$
\begin{cases}u_{t t}-\Delta u=|u|^{p-2} u-|u|^{q-2} u & \text { in }[0, \infty) \times \Omega \\ u=0 & \text { on }[0, \infty) \times \Gamma_{0} \\ \frac{\partial u}{\partial v}=-\alpha(x)\left(\left|u_{t}\right|^{\mu-2} u_{t}+\left|u_{t}\right|^{m-2} u_{t}\right) & \text { on }[0, \infty) \times \Gamma_{1} \\ u(0, x)=u_{0}(x), \quad u_{t}(0, x)=u_{1}(x), & \text { in } \Omega\end{cases}
$$

where $1<\mu \leq m, 1<q<p$, can be treated. When $1<q<2$ the use of a different argument in the proof of local existence is required. The precise assumptions are given later, since they become more shrinking passing from local existence to blowup through global existence results. The organization of the paper is simple: section 2 is concerned with local existence, while section 3 deals with global existence and blow-up results.

2. Local existence. We prove a local existence result for the solutions of (1) which includes Theorem 1 as a particular case. Our assumptions on $Q$ are the following

(Q1) $Q$ is a Carathéodory real function in $\Gamma_{1} \times \mathbb{R}$, and there are a measurable nonnegative function $\alpha$ on $\Gamma_{1}$ and an exponent $m>1$ such that, if $m \geq 2$,

$$
(Q(x, v)-Q(x, w))(v-w) \geq \alpha(x)|v-w|^{m}
$$

for all $x \in \Gamma_{1}, v, w \in \mathbb{R}$, while, if $1<m<2$,

$$
(Q(x, v)-Q(x, w))(v-w) \geq\left.\alpha(x)|| v\right|^{m-2} v-\left.|w|^{m-2} w\right|^{m^{\prime}}
$$

for all $x \in \Gamma_{1}, v, w \in \mathbb{R}$, where $1 / m+1 / m^{\prime}=1$;

(Q2) there are $1<\mu \leq m$ and $c_{1}>0$ such that

$$
|Q(x, v)| \leq c_{1} \alpha(x)\left(|v|^{\mu-1}+|v|^{m-1}\right)
$$

for all $x \in \Gamma_{1}, v \in \mathbb{R}$.

It is clear that

$$
Q_{0}(x, v)=\alpha(x)\left(|v|^{\mu-2} v+|v|^{m-2} v\right), \quad 1<\mu \leq m,
$$

satisfies the assumptions we made on $Q$. Indeed,

$$
\left(Q_{0}(x, v)-Q_{0}(x, w)\right)(v-w) \geq \alpha(x)\left(|v|^{m-2} v-|v|^{m-2} v\right)(v-w) .
$$

Hence, when $m \geq 2$, (Q1) immediately follows from the elementary inequality

$$
\left(|v|^{m-2} v-|w|^{m-2} w\right)(v-w) \geq \text { Const. }|v-w|^{m}, \quad v, w \in \mathbb{R}
$$

where the constant depends only on $m$. When $1<m<2$ the condition (Q1) holds since $m^{\prime}>2$, again with an application of (13) to $|v|^{m-2} v$ and $|w|^{m-2} w$. 
We also remark for a future use some consequences of (Q1)-(Q2). First of all it follows that

$$
Q(x, v) v \geq \alpha(x)|v|^{m}
$$

for all $x \in \Gamma_{1}, v \in \mathbb{R}$, and, moreover, that $Q(x, \cdot)$ is increasing for all $x \in \Gamma_{1}$, and $Q(\cdot, 0) \equiv 0$ by the continuity of $Q$ in the second variable. Then, if we set

$$
\Phi(x, u)=\int_{0}^{u} Q(x, s) d s,
$$

we obtain

$$
\Phi(x, u) \geq \frac{\alpha(x)}{m}|v|^{m} \quad \text { for all } x \in \Gamma_{1}, v \in \mathbb{R} .
$$

Our assumption concerning $f$ is the following

(F1) $f(x, 0)=0$ and there are $p>2$ and $c_{2}>0$ such that

$$
\left|f\left(x, u_{1}\right)-f\left(x, u_{2}\right)\right| \leq c_{2}\left|u_{1}-u_{2}\right|\left(1+\left|u_{1}\right|^{p-2}+\left|u_{2}\right|^{p-2}\right)
$$

for all $x \in \Omega, u_{1}, u_{2} \in \mathbb{R}$.

It is easy to see that

$$
f_{0}(x, u)=a|u|^{q-2} u+b|u|^{p-2} u, \quad 2 \leq q<p, \quad a, b \in \mathbb{R},
$$

satisfies (F1).

We shall also consider later the nonlinearity $f_{0}$ when $1<q<2$. In this case (F1) is not satisfied and the proof of local existence requires the use of a different argument.

Theorem 4. Suppose that (Q1)-(Q2) and (F1) hold, that $2<p \leq 1+2^{*} / 2$, and $u_{0} \in H_{\Gamma_{0}}^{1}(\Omega), u_{1} \in L^{2}(\Omega)$. Then there is $T>0$ and a unique weak solution of (1) in $(0, T) \times \Omega$ such that $(7)-(8)$ hold, with the energy identity

$$
E(t)-E(s)=-\int_{s}^{t} \int_{\Gamma_{1}} Q\left(\cdot, u_{t}\right) u_{t}
$$

for $0 \leq s \leq t$, where

$$
E(t)=\frac{1}{2}\left\|u_{t}(t)\right\|_{2}^{2}+\frac{1}{2}\|\nabla u(t)\|_{2}^{2}-\int_{\Omega} F(\cdot, u(t)),
$$

and

$$
F(x, u)=\int_{0}^{u} f(x, s) d s \quad \text { for } x \in \Omega, u \in \mathbb{R} .
$$

REMARK 4. By a weak solution of (1) we mean a function $u$ (with the required regularity) such that 


$$
\left.\int_{\Omega} u_{t} \varphi\right|_{0} ^{t}=\int_{0}^{t} \int_{\Omega} u_{t} \varphi_{t}-\nabla u \nabla \varphi+f(\cdot, u) \varphi-\int_{0}^{t} \int_{\Gamma_{1}} Q\left(\cdot, u_{t}\right) \varphi
$$

for all $\varphi \in C\left([0, T] ; H_{\Gamma_{0}}^{1}(\Omega)\right) \cap C^{1}\left([0, T] ; L^{2}(\Omega)\right) \cap L^{m}\left((0, T) \times \Gamma_{1}, \alpha\right)$ and $t \in(0, T]$.

An important tool in the proof is the study of the simpler problem

$$
\begin{cases}u_{t t}-\Delta u=g(t, x) & \text { in }[0, T) \times \Omega \\ u=0 & \text { on }[0, T) \times \Gamma_{0} \\ \frac{\partial u}{\partial v}=-Q\left(x, u_{t}\right) & \text { on }[0, T) \times \Gamma_{1} \\ u(0, x)=u_{0}(x), \quad u_{t}(0, x)=u_{1}(x) & \text { on } \Omega\end{cases}
$$

where $T>0$ and $g$ is a fixed forcing term on $(0, T) \times \Omega$.

Lemma 1. Suppose that (Q1)-(Q2) hold, that $g \in W^{1,1}\left(0, T ; L^{2}(\Omega)\right), u_{0}$, $u_{1} \in H_{\Gamma_{0}}^{1}(\Omega)$,

$$
\Delta u_{0} \in L^{2}(\Omega), \quad \frac{\partial u_{0}}{\partial \nu}=-Q\left(\cdot, u_{1}\right) \quad \text { on } \Gamma_{1},
$$

and $u_{1} \in L^{m}\left(\Gamma_{1}, \alpha\right)$. Then (22) has a unique global solution in $(0, T) \times \Omega$ such that

$$
\begin{gathered}
u \in W^{1, \infty}\left(0, T ; H_{\Gamma_{0}}^{1}(\Omega)\right) \cap W^{2, \infty}\left(0, T ; L^{2}(\Omega)\right), \\
u_{t} \in L^{m}\left((0, T) \times \Gamma_{1}, \alpha\right) .
\end{gathered}
$$

REMARK 5. In (23) the Laplacian of $u_{0}$ is taken in distributional sense, and $\left.\frac{\partial u_{0}}{\partial v}\right|_{\Gamma_{1}}$ is not in general well defined by trace theorem, but again in distributional sense, that is $(23)_{2}$ means that

$$
-\int_{\Omega} \Delta u_{0} \varphi=\int_{\Omega} \nabla u_{0} \nabla \varphi+\int_{\Gamma_{1}} Q\left(x, u_{1}\right) \varphi
$$

for all $\varphi \in H_{\Gamma_{0}}^{1}(\Omega) \cap L^{m}\left(\Gamma_{1}, \alpha\right)$ (with a slight abuse of notation). See also [38].

Moreover, by a solution of (22) we mean a function $u$ satisfying (24)-(25) and such that

$$
\int_{\Omega} u_{t t} \varphi+\int_{\Omega} \nabla u \nabla \varphi+\int_{\Gamma_{1}} Q\left(\cdot, u_{t}\right) \varphi=\int_{\Omega} g \varphi
$$

for all $\varphi \in H_{\Gamma_{0}}^{1}(\Omega) \cap L^{m}\left(\Gamma_{1}, \alpha\right)$.

Proof. We apply nonlinear semigroup theory. Let $H=H_{\Gamma_{0}}^{1}(\Omega) \times L^{2}(\Omega)$, endowed with the scalar product

$$
\left\langle\left[\begin{array}{l}
u_{1} \\
v_{1}
\end{array}\right],\left[\begin{array}{l}
u_{2} \\
v_{2}
\end{array}\right]\right\rangle_{H}=\int_{\Omega}\left(\nabla u_{1} \nabla u_{2}+v_{1} v_{2}\right),
$$

and $A: \mathcal{D}(A) \subset H \rightarrow H$ the operator given by 


$$
A\left[\begin{array}{l}
u \\
v
\end{array}\right]=\left[\begin{array}{c}
-v \\
-\Delta u
\end{array}\right]
$$

with

$$
\mathcal{D}(A)=\left\{\left[\begin{array}{l}
u \\
v
\end{array}\right] \in\left[H_{\Gamma_{0}}^{1}(\Omega)\right]^{2}: \Delta u \in L^{2}(\Omega), \quad v \in L^{m}\left(\Gamma_{1}, \alpha\right), \quad \frac{\partial u}{\partial v}=-Q(\cdot, v) \quad \text { on } \Gamma_{1}\right\} .
$$

Then (22) can be written as

$$
\left[\begin{array}{c}
u \\
u_{t}
\end{array}\right]_{t}=A\left[\begin{array}{l}
u \\
u_{t}
\end{array}\right]+\left[\begin{array}{l}
0 \\
g
\end{array}\right], \quad\left[\begin{array}{l}
u \\
u_{t}
\end{array}\right] \in \mathcal{D}(A),
$$

and the existence of $u$ satisfying (22) and (24) can be proved applying [3, Theorem 2.2, p. 131], provided that $A$ is a maximal monotone operator in $H$. The monotonicity of $A$ immediately follows by (Q1) as

$$
\left\langle A\left[\begin{array}{l}
u_{1} \\
v_{1}
\end{array}\right]-A\left[\begin{array}{l}
u_{2} \\
v_{2}
\end{array}\right],\left[\begin{array}{l}
u_{1} \\
v_{1}
\end{array}\right]-\left[\begin{array}{l}
u_{2} \\
v_{2}
\end{array}\right]\right\rangle_{H}=\int_{\Gamma_{1}}\left(Q\left(\cdot, v_{1}\right)-Q\left(\cdot, v_{2}\right)\right)\left(v_{1}-v_{2}\right) .
$$

To prove that $A$ is maximal we shall equivalently prove, by the nonlinear version of Minty's Theorem (see [3, pp. 73-74]), that the equation

$$
A\left[\begin{array}{l}
u \\
v
\end{array}\right]+\left[\begin{array}{l}
u \\
v
\end{array}\right]=h
$$

has a solution $\left[\begin{array}{l}u \\ v\end{array}\right] \in \mathcal{D}(A)$ for every $h=\left[\begin{array}{l}h_{1} \\ h_{2}\end{array}\right] \in H$, i.e. that for every $h_{1} \in H_{\Gamma_{0}}^{1}(\Omega)$ and $h_{2} \in L^{2}(\Omega)$ there are $u, v \in H_{\Gamma_{0}}^{1}(\Omega)$ such that $\Delta u \in L^{2}(\Omega)$ and $v \in L^{m}\left(\Gamma_{1}, \alpha\right)$, which solve

$$
\begin{cases}-v+u=h_{1} & \text { in } \Omega, \\ -\Delta u+v=h_{2} & \text { in } \Omega, \\ \frac{\partial u}{\partial v}=-Q(\cdot, v) & \text { on } \Gamma_{1} .\end{cases}
$$

This is equivalent to find $v \in X=H_{\Gamma_{0}}^{1}(\Omega) \cap L^{m}\left(\Gamma_{1}, \alpha\right)$ such that

$$
\int_{\Omega}(\nabla v \nabla \varphi+v \varphi)+\int_{\Gamma_{1}} Q(\cdot, v) \varphi=\int_{\Omega} h_{2} \varphi-\nabla h_{1} \nabla \varphi
$$

for all $\varphi \in X$. Indeed, if $\left[\begin{array}{l}u \\ v\end{array}\right]$ solves (27), then (28) holds, and, vice-versa, if (28) holds then, taking $\varphi \in C_{c}^{\infty}(\Omega)$, one first obtains that $-\Delta\left(v+h_{1}\right)+v=h_{2}$, so $u=v+h_{1}$ has Laplacian in $L^{2}(\Omega)$ and then (27) follows in a standard way.

The functional

$$
I(v)=\frac{1}{2}\|\nabla v\|_{2}^{2}+\frac{1}{2}\|v\|_{2}^{2}+\int_{\Gamma_{1}} \Phi(\cdot, v)-\int_{\Omega}\left(h_{2} v-\nabla h_{1} \nabla v\right)
$$


(we recall that $\Phi$ was defined on $(15)$ ) is of class $C^{1}(X, \mathbb{R})$ by $(\mathrm{Q} 2)$ and coercive by (16). By the direct method of the Calculus of Variations (see for example [33]), there is a critical point of $I$, namely a solution of (28).

We proved the existence of $u$ satisfying (22) and (24), so to complete the proof we have only to verify (25), which is not a direct consequence of (24) or of the property that $u_{t}(t) \in L^{m}\left(\Gamma_{1}, \alpha\right)$ for all $t \in[0, T]$. By (24) and the trace theorem one obtains in particular that $u_{t} \in L^{2}\left(0, T ; L^{2}\left(\Gamma_{1}\right)\right)$; hence, by the Riesz Theorem, $u_{t}$ has a representative in $L^{2}\left((0, T) \times \Gamma_{1}\right)$. Since $Q$ is a Carathéodory function also $Q\left(\cdot, u_{t}\right)$ is measurable on $(0, T) \times \Gamma_{1}$. By (24) we can derive from (26) the energy identity

$$
\frac{d}{d t}\left(\frac{1}{2}\|\nabla u(t)\|_{2}^{2}+\frac{1}{2}\left\|u_{t}(t)\right\|_{2}^{2}\right)+\int_{\Gamma_{1}} Q\left(\cdot, u_{t}(t)\right) u_{t}(t)=\int_{\Omega} g(t) u_{t}(t) .
$$

Integrating (29) and using (14) and (24) we get $v \in L^{m}\left((0, T) \times \Gamma_{1}\right)$, concluding the proof.

We are now ready to give the

Proof of Theorem 4. We adapt the ideas of [11, Section 2], using Lemma 1 instead of [26, Theorem 3.1, Chapter 1]. The only changes arise in proving the analogous of [11, Proposition 2.1], which can be formulated for (1) as follows. Given any

$$
u \in X_{T}=C\left([0, T] ; H_{\Gamma_{0}}^{1}(\Omega)\right) \cap C^{1}\left([0, T] ; L^{2}(\Omega)\right)
$$

there is a unique weak solution $v \in X_{T}$ (in the sense of Remark 4) of

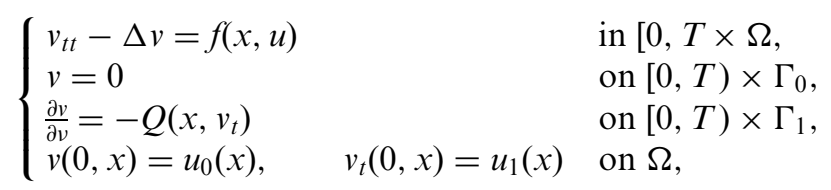

such that $v_{t} \in L^{m}\left((0, T) \times \Gamma_{1}, \alpha\right)$ and the energy identity

$$
\frac{1}{2}\left\|v_{t}\right\|_{2}^{2}+\left.\frac{1}{2}\|\nabla v\|_{2}^{2}\right|_{s} ^{t}+\int_{s}^{t} \int_{\Gamma_{1}} Q\left(\cdot, v_{t}\right) v_{t}=\int_{s}^{t} \int_{\Omega} f(\cdot, u) v_{t}
$$

holds for $0 \leq s \leq t$.

We start approximating the data $u_{0}$ and $u_{1}$, respectively in $H_{\Gamma_{0}}^{1}(\Omega)$ and $L^{2}(\Omega)$, with more regular (enough to apply Lemma 1 ) data $u_{0}^{k}, u_{1}^{k}, k \in \mathbb{N}$, verifying the compatibility condition $(23)_{2}$.

We simply approximate $u_{1}$ in $L^{2}(\Omega)$ with $u_{1}^{k} \in C_{c}^{\infty}(\Omega)$, so that, to satisfy also (23) we must find $u_{0}^{k} \in H_{\Gamma_{0}}^{1}(\Omega)$ such that $\Delta u_{0}^{k} \in L^{2}(\Omega), \frac{\partial u_{0}^{k}}{\partial \nu}=0$ on $\Gamma_{1}$ and $u_{0}^{k} \rightarrow u_{0}$ in $H_{\Gamma_{0}}^{1}(\Omega)$. This is equivalent to prove that the linear subspace

$$
X=\left\{v \in H_{\Gamma_{0}}^{1}(\Omega): \Delta v \in L^{2}(\Omega), \quad \frac{\partial v}{\partial v}=0 \quad \text { on } \Gamma_{1}\right\}
$$

is dense in $H_{\Gamma_{0}}^{1}(\Omega)$, i.e., thanks to the Hahn-Banach theorem, that for any given $u \in H_{\Gamma_{0}}^{1}(\Omega)$ such that 


$$
\langle u, v\rangle_{\Gamma_{\Gamma_{0}}^{1}(\Omega)}=0 \quad \text { for all } v \in X,
$$

implies that $u=0$. This fact can be proved with the following simple argument: given any such $u$, take $v$ such that

$$
\langle v, \phi\rangle_{H_{\Gamma_{0}}^{1}(\Omega)}=\langle u, \phi\rangle_{L^{2}(\Omega)} \quad \text { for all } \phi \in \mathrm{H}_{\Gamma_{0}}^{1}(\Omega),
$$

which exists by Lax-Milgram theorem (see [4]). Then $\Delta v=u \in L^{2}(\Omega)$ and $\frac{\partial v}{\partial v}=0$ (in the sense of Remark 5), so $v \in X$. Putting $\phi=u$ in (32) and using (31) we obtain that $u=0$.

Next we approximate $u$ in $C\left([0, T] ; H_{\Gamma_{0}}^{1}(\Omega)\right) \cap C^{1}\left([0, T] ; L^{2}(\Omega)\right)$, endowed with the standard norm $\|u\|=\max _{t \in[0, T]}\left\|u_{t}(t)\right\|_{2}+\|u(t)\|_{H^{1}(\Omega)}$, with $u^{k} \in C^{\infty}([0, T] \times \bar{\Omega})$, by standard convolution arguments (see $[4])$. We note that $f(x, \cdot) \in \operatorname{Lip}_{\text {loc }}(\mathbb{R})$ and

$$
\left|\frac{\partial f}{\partial u}(x, u)\right| \leq 2 c_{2}\left(1+|u|^{p-2}\right) .
$$

by (F1). Consequently $f\left(\cdot, u^{k}\right) \in W^{1,1}\left(0, T ; L^{2}(\Omega)\right)$ by (F1)and (33). Then, applying Lemma 1 , for any $k \in \mathbb{N}$ there is

$$
v^{k} \in W^{1, \infty}\left(0, T ; H_{\Gamma_{0}}^{1}(\Omega)\right) \cap W^{2, \infty}\left(0, T ; L^{2}(\Omega)\right), \quad v_{t}^{k} \in L^{m}\left((0, T) \times \Gamma_{1}, \alpha\right),
$$

solution of

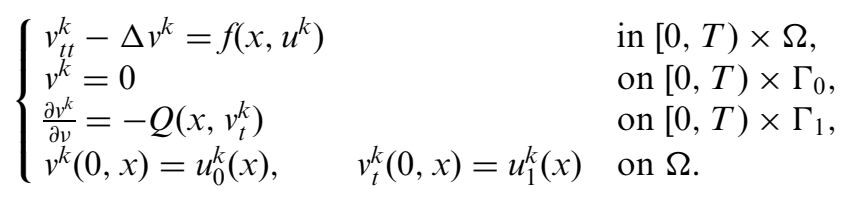

Now we verify that $v^{k}$ is a Cauchy sequence in $X_{T}$, reproducing verbatim the proof of [11, Proposition 2.1] and using (F1) and (Q1).

To verify that (up to a subsequence) $v_{t}^{k}$ is a Cauchy sequence in $L^{m}\left(\Gamma_{1}, \alpha\right)$, we note that, using the same arguments of [11], one can deduce that

$$
\int_{0}^{T} \int_{\Gamma_{1}}\left(Q\left(x, v_{t}^{j}\right)-Q\left(x, v_{t}^{k}\right)\right)\left(v_{t}^{j}-v_{t}^{k}\right) \rightarrow 0 \quad \text { as } j, k \rightarrow \infty .
$$

When $m \geq 2$, (Q1) immediately yields our claim. When $1<m<2$ from (Q1) it follows that

$$
\left\|\left|v_{t}^{j}\right|^{m-2} v_{t}^{j}-\left|v_{t}^{k}\right|^{m-2} v_{t}^{k}\right\|_{L^{m^{\prime}}\left(\Gamma_{1}, \alpha\right)} \rightarrow 0 \quad \text { as } j, k \rightarrow \infty
$$

so, using [4, Théorème IV.9], there is $\chi \in L^{m^{\prime}}\left(\Gamma_{1}, \alpha\right)$ such that (up to a subsequence) $\left|v_{t}^{k}\right|^{m-1} \leq \chi$ on $\Gamma_{1}$ and $v_{t}^{k}$ is $\mu_{\alpha}$-a.e. convergent on $\Gamma_{1}$. Then, by the Lebesgue dominated convergence theorem, $v_{t}^{k}$ is convergent in $L^{m}\left(\Gamma_{1}, \alpha\right)$.

Passing to the limit as $k \rightarrow 0$ in (34), and using (F1) and (Q2), we find a unique solution of (30) exactly as in [11], concluding the proof. 
As mentioned above, assumption (F1) is not satisfied by $f_{0}$ given in (17) when $1<q<2$ (unless $a=0$ ). The rest of this section is devoted to this case. Indeed we shall consider nonlinearities $f$ satisfying the more general assumption

(F2) $f(x, 0)=0$ and there are $1<q<2<p$ and $c_{2}>0$ such that

$$
\left|f\left(x, u_{1}\right)-f\left(x, u_{2}\right)\right| \leq c_{2}\left[\left|u_{1}-u_{2}\right|\left(1+\left|u_{1}\right|^{p-2}+\left|u_{2}\right|^{p-2}\right)+\left|u_{1}-u_{2}\right|^{q-1}\right]
$$

for all $x \in \Omega, u_{1}, u_{2} \in \mathbb{R}$.

This case requires a different technique in order to prove local existence of the solution, that is the application of the Schauder fixed point theorem instead that the contraction principle as in [11]. For this reason we cannot consider the case $p=1+2^{*} / 2$ and we renounce to any claim of uniqueness. This is the content of

Theorem 5. Suppose that (Q1)-(Q2) and (F2) hold, and $2<p<1+2 * / 2$. Then the statement of Theorem 4 (except for the uniqueness of the solution founded) holds.

Proof. We indicate the modifications of the proof of Theorem 4 which are needed. Certainly we cannot directly use Lemma 1 , since $f\left(\cdot, u^{k}\right)$ does not belong in general to $W^{1,1}\left(0, T ; L^{2}(\Omega)\right)$, in order to prove that (34) has a solution for any $k \in \mathbb{N}$. We shall use a further simple approximation process. Indeed by (F2) $f\left(\cdot, u^{k}\right) \in L^{2}((0, T) \times \Omega)$, and then we can choose $\chi^{k, \varepsilon} \in C_{c}^{\infty}((0, T) \times \Omega), \varepsilon>0$, such that $\chi^{k, \varepsilon} \rightarrow f\left(\cdot, u^{k}\right)$ in $L^{2}((0, T) \times \Omega)$ as $\varepsilon \rightarrow 0^{+}$. By Lemma 1 there is $v^{k, \varepsilon} \in W^{1, \infty}\left(0, T ; H_{\Gamma_{0}}^{1}(\Omega)\right) \cap W^{2, \infty}\left(0, T ; L^{2}(\Omega)\right)$ solution of (22) with $g=\chi^{k, \varepsilon}$. Then $w=v^{k, \varepsilon}-v^{k, \delta}, \varepsilon, \delta>0$, is a solution of

$$
\begin{cases}w_{t t}-\Delta w=\chi^{k, \varepsilon}-\chi^{k, \delta} & \text { in }[0, T) \times \Omega, \\ w=0 & \text { on }\left[0, T \times \Gamma_{0},\right. \\ \frac{\partial w}{\partial v}=-Q\left(x, v_{t}^{k, \varepsilon}\right)+Q\left(x, v_{t}^{k, \delta}\right) & \text { on }[0, T) \times \Gamma_{1}, \\ w(0, x)=w_{t}(0, x)=0 & \text { on } \Omega,\end{cases}
$$

which verifies the energy identity

$$
\begin{aligned}
& \frac{1}{2}\left\|w_{t}(t)\right\|_{2}^{2}+\frac{1}{2}\|\nabla w(t)\|_{2}^{2}+\int_{0}^{t} \int_{\Gamma_{1}}\left(Q\left(x, v_{t}^{k, \varepsilon}\right)-Q\left(x, v_{t}^{k, \delta}\right)\right)\left(v_{t}^{k, \varepsilon}-v_{t}^{k, \delta}\right) \\
= & \int_{0}^{t} \int_{\Omega}\left(\chi^{k, \varepsilon}-\chi^{k, \delta}\right) w_{t} .
\end{aligned}
$$

Then, by (Q1), a standard use of the Gronwall lemma shows that $\left(v^{k, \varepsilon}\right)_{\varepsilon}$ is a Cauchy sequence in $C\left([0, T], H_{\Gamma_{0}}^{1}(\Omega)\right) \cap C^{1}\left([0, T] ; L^{2}(\Omega)\right)$. Hence, using the same arguments of the proof of Theorem 4, we prove that $\left(v_{t}^{k, \varepsilon}\right)_{\varepsilon}$ is a Cauchy sequence in $L^{m}\left(\left(0, T \times \Gamma_{1}, \alpha\right)\right.$ and the limit $v^{k}$ (as $\left.\varepsilon \rightarrow 0\right)$ solves (34). Moreover, starting from the energy identities for $v^{k, \varepsilon}$ and for $v^{j, \varepsilon}-v^{k, \varepsilon}$ one obtains that $v^{k}$ and $v^{j}-v^{k}$ satisfy the energy identity.

To show here that $v^{k}$ is a Cauchy sequence in $X_{T}$ we have to modify the previous arguments first given in [11], and use a more general a priori estimate. Indeed, we 
shall establish an estimate which is stronger than what is immediately needed here. By (F2)

$$
\begin{aligned}
& I_{0}:=\left|\int_{\Omega}\left(f\left(\cdot, u_{1}\right)-f\left(\cdot, u_{2}\right)\right)\left(v_{1}-v_{2}\right)\right| \\
& \leq c_{2} \int_{\Omega}\left[\left|u_{1}-u_{2}\right|\left(1+\left|u_{1}\right|^{p-2}+\left|u_{2}\right|^{p-2}\right)+\left|u_{1}-u_{2}\right|^{q-1}\right]\left|v_{1}-v_{2}\right|
\end{aligned}
$$

for any $u_{1}, u_{2} \in H_{\Gamma_{0}}^{1}(\Omega), v_{1}, v_{2} \in L^{2}(\Omega)$. Fix a number $r \in\left(2 p-2,2^{*}\right)$, so that $p<1+r / 2<1+2 * / 2$. As $2(q-1)<2<r$, using Hölder inequality

$$
I_{0} \leq C\left[\left\|u_{1}-u_{2}\right\|_{r}\left(1+\left\|u_{1}\right\|_{s(p-2)}^{p-2}+\left\|u_{2}\right\|_{s(p-2)}^{p-2}\right)+\left\|u_{1}-u_{2}\right\|_{r}^{q-1}\right]\left\|v_{1}-v_{2}\right\|_{2}
$$

where

$$
\frac{1}{r}+\frac{1}{2}+\frac{1}{s}=1
$$

(here and in the sequel of the proof $C=C\left(p, q, \Omega, T, u_{0}, u_{1}\right)$ will denote positive constants, possibly different). Then, since $s(p-2) \leq r$, using Sobolev embedding, we obtain

$$
I_{0} \leq C\left[\left\|u_{1}-u_{2}\right\|_{r}\left(1+\left\|\nabla u_{1}\right\|_{2}^{p-2}+\left\|\nabla u_{2}\right\|_{2}^{p-2}\right)+\left\|u_{1}-u_{2}\right\|_{r}^{q-1}\right]\left\|v_{1}-v_{2}\right\|_{2} .
$$

Actually, to prove that $v^{k}$ is a Cauchy sequence in $X_{T}$ it is enough to apply (36) in the weaker form (since $r<2^{*}$ )

$$
I_{0} \leq C\left[\left\|\nabla u_{1}-\nabla u_{2}\right\|_{2}\left(1+\left\|\nabla u_{1}\right\|_{2}^{p-2}+\left\|\nabla u_{2}\right\|_{2}^{p-2}\right)+\left\|\nabla u_{1}-\nabla u_{2}\right\|_{2}^{q-1}\right]\left\|v_{1}-v_{2}\right\|_{2}
$$

to $u_{1}=u^{j}, u_{2}=u^{k}, v_{1}=v_{t}^{j}, v_{2}=v_{t}^{k}$ and argue as in [11]. Then we can conclude the proof of the existence of a solution of (30) as in Theorem 4.

The third and main modification in the proof of Theorem 4 is that, once we proved the analogous of [11, Proposition 2.1], we cannot follow verbatim [11, Proof of Theorem 2.1]. Indeed, this was exactly the application of the contraction principle which, as mentioned above, is no longer appropriate here.

We argue as follows. Define

$$
Y_{T}=\left\{u \in X_{T}: u(0)=u_{0}, u_{t}(0)=u_{1}\right\},
$$

and $\Psi: Y_{T} \rightarrow Y_{T}$ by $\Psi(u)=v$ where $v$ solves (30). We can still easily prove that, for $R$ sufficiently large and $T$ sufficiently small, $\Psi$ maps the ball $B_{R}$ of $Y_{T}$ in itself. Indeed (37) yields that, for $u \in B_{R}$ and $R \geq 1$ (since $q<2<p$ )

$$
\left|\int_{\Omega} f(\cdot, u) v_{t}\right| \leq C\left(\|\nabla u\|_{2}+\|\nabla u\|_{2}^{p-1}+\|\nabla u\|_{2}^{q-1}\right)\left\|v_{t}\right\|_{2} \leq 3 C R^{p-1}\left\|v_{t}\right\|_{2},
$$

so that we can argue as in [11] using the energy identity. 
Next we apply to $\Psi$ the Schauder fixed point theorem (see [10, Corollary 3.6.2]). We argue as follows: given any sequence $u^{k}$ in $B_{R}$, denoting $w=u^{k}-u^{j}$ for $k, j \in \mathbb{N}$, $w$ solves the problem

$$
\begin{cases}w_{t t}-\Delta w=f\left(x, u^{j}\right)-f\left(x, u^{k}\right) & \text { in }[0, T) \times \Omega \\ w=0 & \text { on }\left[0, T \times \Gamma_{0},\right. \\ \frac{\partial w}{\partial v}=-Q\left(x, v_{t}^{j}\right)+Q\left(x, v_{t}^{k}\right) & \text { on }[0, T) \times \Gamma_{1}, \\ w(0, x)=u_{0}^{j}(x)-u_{0}^{k}(x), \quad w_{t}(0, x)=u_{1}^{j}(x)-u_{1}^{k}(x) & \text { on } \Omega .\end{cases}
$$

Hence, by the energy identity and (Q1)

$$
\begin{aligned}
& I_{1}:=\frac{1}{2}\left\|w_{t}(t)\right\|_{2}^{2}+\frac{1}{2}\|\nabla w(t)\|_{2}^{2} \leq \frac{1}{2}\left\|w_{t}(0)\right\|_{2}^{2}+\frac{1}{2}\|\nabla w(0)\|_{2}^{2} \\
& +\int_{0}^{t} \mid \int_{\Omega}\left(f\left(\cdot, u^{j}\right)-f\left(\cdot, u^{k}\right)\left(v_{t}^{j}-v_{t}^{k}\right) \mid .\right.
\end{aligned}
$$

By (36), which essentially depends on the assumption $p<1+2 * / 2$, again with $R \geq 1$

$$
\begin{aligned}
I_{1} & \leq \frac{1}{2}\left\|u_{1}^{j}-u_{1}^{k}\right\|_{2}^{2}+\frac{1}{2}\left\|\nabla u_{0}^{j}-\nabla u_{0}^{k}\right\|_{2}^{2} \\
& +C\left[3 R^{p-2}\left\|u^{j}(t)-u^{k}(t)\right\|_{r}+\left\|u^{j}(t)-u^{k}(t)\right\|_{r}^{q-1}\right]\left\|w_{t}(t)\right\|_{2} \\
& \leq \frac{1}{2}\left\|u_{1}^{j}-u_{1}^{k}\right\|_{2}^{2}+\frac{1}{2}\left\|\nabla u_{0}^{j}-\nabla u_{0}^{k}\right\|_{2}^{2} \\
& +C\left(3 R^{p-2}\left\|u^{j}-u^{k}\right\|_{C\left([0, T] ; L^{r}(\Omega)\right)}+\left\|u^{j}-u^{k}\right\|_{C\left([0, T] ; L^{r}(\Omega)\right)}^{q-1}\right)\left\|w_{t}(t)\right\|_{2} .
\end{aligned}
$$

Now we claim that, up to a subsequence,

$$
\left\|u^{j}-u^{k}\right\|_{C\left([0, T] ; L^{r}(\Omega)\right)} \rightarrow 0 \quad \text { as } \mathrm{j}, \mathrm{k} \rightarrow \infty .
$$

Indeed $\left\{u^{k}(t)\right\}_{k}$ is relatively compact in $L^{r}(\Omega)$ for all $t \in[0, T]$ by the Rellich-Kondrachov theorem. Moreover, for all $s, t \in[0, T]$, using the interpolation inequality, the Sobolev embedding and the boundness of $u^{k}$ in $Y_{T}$

$$
\left\|u^{k}(s)-u^{k}(t)\right\|_{r} \leq\left\|u^{k}(s)-u^{k}(t)\right\|_{2}^{\theta}\left\|u^{k}(s)-u^{k}(t)\right\|_{2^{*}}^{1-\theta} \leq \text { Const. }\left\|u^{k}(s)-u^{k}(t)\right\|_{2}^{\theta}
$$

where (consider for simplicity the case $n \geq 3$ )

$$
\frac{1}{r}=\frac{\theta}{2}+\frac{1-\theta}{2^{*}}
$$

so $\left(u^{k}\right)_{k}$ is equicontinuous in $C\left([0, T] ; L^{r}(\Omega)\right)$. The claim then follows by Ascoli's theorem (see [31]). By using (38) and (39) and a standard argument we prove that $v^{k}$ converges in $B_{R}$, namely the compactness of $\Psi$. At this point we complete the proof as in [11].

3. Global existence and blow-up. This section is devoted to proving our main global existence and blow-up theorems, which include Theorems 2 and 3 as particular cases. For this purpose we use a further assumption on $f$; that is 
(F3) there is $c_{3}>0$ such that

$$
F(x, u) \leq \frac{c_{3}}{p}|u|^{p}
$$

for all $x \in \Omega$ and $u \in \mathbb{R}$, where $F$ is the primitive of $f$ defined in (20).

It is clear that $f_{0}$ given in (17) satisfies (F1)-(F3) when $1<q<p, p>2, a \leq 0$ and $b \in \mathbb{R}$.

We set

$$
K_{0}=\sup _{u \in H_{\Gamma_{0}}^{1}(\Omega), u \neq 0} \frac{\int_{\Omega} F(\cdot, u)}{\|\nabla u\|_{2}^{p}} .
$$

We can suppose without loss of generality that $K_{0}>0$. If this is not the case it is easy to see that $F(x, u) \leq 0$ a.e. in $(0, T) \times \mathbb{R}$, and in this case global existence for arbitrary initial data is an immediate consequence of the local existence, the energy identity and the standard continuation principle. By $(\mathrm{F} 3)$ one can estimate $K_{0} \leq\left(c_{3} B_{1} / p\right)^{1 / p}$, where $B_{1}$ is the optimal constant of the Sobolev embedding $H_{\Gamma_{0}}^{1}(\Omega) \hookrightarrow L^{p}(\Omega)$.

We denote

$$
\lambda_{1}=\left(p / K_{0}\right)^{1 /(p-2)}, \quad E_{1}=\left(\frac{1}{2}-\frac{1}{p}\right) \lambda_{1}^{2} .
$$

REMARK 6. When $f(x, u)=|u|^{p-2} u$ (see [36]) the number $E_{1}$ above is equal to $d$ defined in (4) and also equal to the Mountain Pass level associated to the elliptic problem

$$
\begin{cases}-\Delta u=|u|^{p-2} u & \text { in } \Omega \\ u=0 & \text { on } \Gamma_{0} \\ \frac{\partial u}{\partial \nu}=0 & \text { on } \Gamma_{1}\end{cases}
$$

that is $E_{1}$ is equal to the number $\inf _{\gamma \in \Lambda} \sup _{t \in[0,1]} J(\gamma(t))$ (see (2) for the definition of $J$ ), where

$$
\Lambda=\left\{\gamma \in C\left([0,1] ; H_{\Gamma_{0}}^{1}(\Omega): \gamma(0)=0, \quad J(\gamma(1))<0\right\} .\right.
$$

We begin with a lemma, which illustrates the two situations in which we have information about the time behavior of the solutions of (1).

Lemma 2. Suppose that the assumptions of Theorem 4 (or of Theorem 5) hold, together with (F3), and let $u$ be a solution of (1). Assume moreover that $E(0)<E_{1}$.

(i) If $\left\|\nabla u_{0}\right\|_{2}<\lambda_{1}$ then $\|\nabla u(t)\|_{2}<\lambda_{1}$ for all $t \in[0, T]$.

(ii) If $\left\|\nabla u_{0}\right\|_{2}>\lambda_{1}$ then there is $\lambda_{2}>\lambda_{1}$ such that $\|\nabla u(t)\|_{2} \geq \lambda_{2}$ and $\|u(t)\|_{p} \geq\left(p K_{0} / c_{3}\right)^{1 / p} \lambda_{2}$ for all $t \in[0, T]$.

Proof. We shall omit for simplicity the explicit dependence on time. We first note that, by (40),

$$
E(t) \geq \frac{1}{2}\|\nabla u\|_{2}^{2}-K_{0}\|\nabla u\|_{2}^{p}=g(\|\nabla u\|)
$$


where $g(\lambda)=\lambda^{2} / 2-K_{0} \lambda^{p}, \lambda>0$. Of course, $g$ is increasing for $0<\lambda<\lambda_{1}$, decreasing for $\lambda>\lambda_{1}, g\left(\lambda_{1}\right)=E_{1}$ and $g(\lambda) \rightarrow-\infty$ as $\lambda \rightarrow \infty$. Then, since $E_{0}:=E(0)<E_{1}$, there are $\lambda_{2}^{\prime}<\lambda_{1}<\lambda_{2}$ such that $g\left(\lambda_{2}\right)=g\left(\lambda_{2}^{\prime}\right)=E_{0}$. Denote $\lambda_{0}=\left\|\nabla u_{0}\right\|_{2}$. Also, by (18),

$$
E(t) \leq E_{0} \quad \text { for all } \mathrm{t} \in[0, \mathrm{~T}]
$$

Consider now the case (i), that is $\lambda_{0}<\lambda_{1}$. Then, as by (41) we have $g\left(\lambda_{0}\right) \leq E_{0}$, it follows that $\lambda_{0} \leq \lambda_{2}^{\prime}$. We claim that $\|\nabla u(t)\|_{2} \leq \lambda_{2}^{\prime}$ for all $t \in[0, T]$, so that (i) follows. Suppose, by contradiction, that $\left\|\nabla u\left(t_{0}\right)\right\|_{2}>\lambda_{2}^{\prime}$ for some $t_{0}>0$. By the continuity of $\|\nabla u(\cdot)\|_{2}$ we can suppose that $\left\|\nabla u\left(t_{0}\right)\right\|_{2}<\lambda_{1}$. Then, by $(41), E\left(t_{0}\right) \geq$ $g\left(\left\|\nabla u\left(t_{0}\right)\right\|_{2}\right)>g\left(\lambda_{2}^{\prime}\right)=E_{0}$, in contradiction with (42).

To prove (ii) we deduce as before that $\lambda_{0}>\lambda_{1}$ implies $\|\nabla u(t)\|_{2} \geq \lambda_{2}$ for $t \in[0, T]$. Hence, by (19), (42) and (F3)

$$
\frac{1}{2}\|\nabla u\|_{2}^{2}+\frac{1}{2}\left\|u_{t}\right\|_{2}^{2} \leq E_{0}+\frac{c_{3}}{p}\|u\|_{p}^{p}
$$

and then

$$
\frac{c_{3}}{p}\|u\|_{p}^{p} \geq \frac{1}{2}\|\nabla u\|_{2}^{2}-E_{0} \geq \frac{1}{2} \lambda_{2}^{2}-g\left(\lambda_{2}\right)=K_{0} \lambda_{2}^{p},
$$

by definition of $g$. Finally, $\|u\|_{p} \geq\left(p K_{0} / c_{3}\right)^{1 / p} \lambda_{2}$, which concludes the proof.

Our global existence theorem is the following

Theorem 6. Suppose that the assumptions of Theorem 4 (or of Theorem 5) hold, together with (F3), and that

$$
\left\|\nabla u_{0}\right\|_{2}<\lambda_{1}, \quad E(0)<E_{1} .
$$

Then the solution of (1) is global on $[0, \infty)$, and $\|\nabla u(t)\|_{2} \leq \lambda_{1}$ for all $t \geq 0$.

Proof. It is sufficient to combine the local existence of the solution with the standard continuation principle (see [32]) and the estimate given in Lemma 2-(i).

Now we turn to our blow-up result. We need two further assumptions on the nonlinearities involved:

(Q3) there is $c_{4}>0$ such that

$$
Q(x, v) v \geq c_{4} \alpha(x)\left(|v|^{\mu}+|v|^{m}\right), \quad 1<\mu \leq m,
$$

for all $x \in \Gamma_{1}, v \in \mathbb{R}$;

(F4) there is $\varepsilon_{0}>0$ such that for all $\varepsilon \in\left(0, \varepsilon_{0}\right]$ there exists $c_{5}=c_{5}(\varepsilon)>0$ such that

$$
f(x, u) u-(p-\varepsilon) F(x, u) \geq c_{5}|u|^{p}
$$

for all $x \in \Omega, u \in \mathbb{R}$. 
Remark 7. Clearly $Q_{0}$ given in (12) satisfies (Q3) with $c_{4}=1$, and $f_{0}$ given in (17) satisfies (F4) when $a \leq 0$ and $b>0$, with $\varepsilon_{0}=p-q>0$ and $c_{5}(\varepsilon)=b \varepsilon / p$. Moreover (Q3) immediately follows from (14) when $m=\mu$, while is not a consequence of (Q1)-(Q2) when $\mu<m$. Next (F4) implies the standard growth condition

$$
f(x, u) u \geq p F(x, u) \quad \text { for all } x \in \Omega, u \in \mathbb{R} .
$$

Clearly (F1)-(F3) and (44) are not sufficient to prove a blow-up theorem, since the case $f \equiv 0$ is included and did not produce blow-up.

Theorem 7. Suppose that the assumptions of Theorem 4 (Theorem 5) hold, with (F3)-(F4) and (Q3), that $\alpha \in L^{\infty}\left(\Gamma_{1}\right)$ and that (11) holds. Let $u$ be the solution of (1) given in Theorem 4 (Theorem 5). Then, if

$$
\left\|\nabla u_{0}\right\|_{2}>\lambda_{1}, \quad E(0)<E_{1},
$$

there is $T_{\max }>0$ such that $\|u(t)\|_{p} \rightarrow \infty$ (and so also $\|u(t)\|_{\infty} \rightarrow \infty$ and $\left.\|\nabla u(t)\|_{2} \rightarrow \infty\right)$ as $t \rightarrow T_{\max }^{-}$.

Proof. We appropriately modify the proof of [35, Theorem 2] (see also [11] and [25]). It is enough to prove that no global solution in $[0, \infty)$ can exist. Indeed, the local existence of solutions together with the standard continuation principle (see [32]) yields the blow-up of $\left\|u_{t}(t)\right\|_{2}^{2}+\|\nabla u(t)\|_{2}^{2}$, which, by (43), gives the theorem. Hence assume by contradiction that $u$ be defined on the whole $[0, \infty)$.

Keeping the notations of Lemma 2, let $E_{2} \in\left(E_{0}, E_{1}\right)$ be fixed, and set $\mathcal{H}(t)=E_{2}-E(t)$. Being $\mathcal{H}$ increasing by (18), it follows that

$$
\mathcal{H}(t) \geq \mathcal{H}_{0}:=\mathcal{H}(0)=E_{2}-E_{0}>0 .
$$

We shall omit, for simplicity, explicit dependence on time on the notations. By Lemma 2-(ii),

$$
\mathcal{H}(t) \leq E_{2}-\frac{1}{2}\|\nabla u\|_{2}^{2}+\int_{\Omega} F(\cdot, u) \leq E_{1}-\frac{1}{2} \lambda_{1}^{2}+\int_{\Omega} F(\cdot, u)
$$

and then, by the definition of $E_{1}$ and (F4),

$$
\mathcal{H}(t) \leq \int_{\Omega} F(\cdot, u) \leq \frac{c_{3}}{p}\|u\|_{p}^{p} .
$$

Now we claim that there are positive constants $c_{6}$ and $c_{7}$ such that

$$
I_{2}:=\frac{d}{d t} \int_{\Omega} u_{t} u \geq 2\left\|u_{t}\right\|_{2}^{2}+c_{6}\|u\|_{p}^{p}+c_{7}\|\nabla u\|_{2}^{2}+2 \mathcal{H}-\int_{\Gamma_{1}} Q\left(\cdot, u_{t}\right) u
$$

on $[0, \infty)$. Taking $\varphi=u$ in $(21)$ we obtain

$$
I_{2}=\left\|u_{t}\right\|_{2}^{2}-\|\nabla u\|_{2}^{2}+\int_{\Omega} f(\cdot, u) u-\int_{\Gamma_{1}} Q\left(\cdot, u_{t}\right) u
$$

and then, using (F4), for $0<\varepsilon<\min \left\{\varepsilon_{0}, p-2\right\}$, 


$$
\begin{aligned}
I_{2} & \geq 2\left\|u_{t}\right\|_{2}^{2}+\int_{\Omega}[f(\cdot, u) u-(p-\varepsilon) F(\cdot, u)]+\frac{1}{2}(p-\varepsilon-2)\|\nabla u\|_{2}^{2}-(p-\varepsilon) E_{2} \\
+ & (p-\varepsilon) \mathcal{H}-\int_{\Gamma_{1}} Q\left(\cdot, u_{t}\right) u \\
& \geq 2\left\|u_{t}\right\|_{2}^{2}+c_{5}\|u\|_{p}^{p}+\frac{1}{2}(p-\varepsilon-2)\|\nabla u\|_{2}^{2}-(p-\varepsilon) E_{2}+2 \mathcal{H}-\int_{\Gamma_{1}} Q\left(\cdot, u_{t}\right) u .
\end{aligned}
$$

By Lemma 2-(ii)

$$
\frac{1}{2}(p-\varepsilon-2)\|\nabla u\|_{2}^{2}-(p-\varepsilon) E_{2} \geq c_{7}\|\nabla u\|_{2}^{2}+c_{8},
$$

where

$$
c_{7}=c_{7}(\varepsilon)=\frac{1}{2}(p-\varepsilon-2)\left(1-\lambda_{1}^{2} / \lambda_{2}^{2}\right), \quad c_{8}=c_{8}(\varepsilon)=\frac{1}{2}(p-\varepsilon-2) \lambda_{1}^{2}-(p-\varepsilon) E_{2} .
$$

Clearly $c_{7}>0$ and, as $\varepsilon \rightarrow 0^{+}$,

$$
c_{8}(\varepsilon) \rightarrow \frac{1}{2}(p-2) \lambda_{1}^{2}-p E_{2}>\frac{1}{2}(p-2) \lambda_{1}^{2}-p E_{1}=0,
$$

so also $c_{8}(\varepsilon)>0$ for $\varepsilon$ sufficiently small. Fixing a sufficiently small $\varepsilon$ and setting $c_{6}=c_{5}(\varepsilon)$ we conclude the proof of (47).

Now, in order to use the technique of [11] and [25], we estimate the last term in (47). We denote

$$
\|\cdot\|_{l, \Gamma_{1}}=\|\cdot\|_{L^{l}\left(\Gamma_{1}\right)}, \quad\|\cdot\|_{l, \Gamma_{1}, \alpha}=\|\cdot\|_{L^{l}\left(\Gamma_{1}, \alpha\right)}, \quad \text { for any } l>1 .
$$

Using (Q2), the Hölder inequality (with respect to $\mu_{\alpha}$ ), and the fact that $\alpha \in L^{\infty}\left(\Gamma_{1}\right)$,

$$
I_{3}:=\left|\int_{\Gamma_{1}} Q\left(\cdot, u_{t}\right) u\right| \leq C_{1}\left(\left\|u_{t}\right\|_{\mu, \Gamma_{1}, \alpha}^{\mu-1}\|u\|_{\mu, \Gamma_{1}}+\left\|u_{t}\right\|_{m, \Gamma_{1}, \alpha}^{m-1}\|u\|_{m, \Gamma_{1}}\right)
$$

with $C_{1}=c_{1}\left(\|\alpha\|_{\infty}^{1 / \mu}+1\right)$. Applying the Hölder inequality again, since $\mu \leq m$ we obtain

$$
I_{3} \leq C_{2}\left(\left\|u_{t}\right\|_{\mu, \Gamma_{1}, \alpha}^{\mu-1}+\left\|u_{t}\right\|_{m, \Gamma_{1}, \alpha}^{m-1}\right)\|u\|_{m, \Gamma_{1}}
$$

with $C_{2}=C_{2}\left(\mu, m, c_{1},\|\alpha\|_{\infty}, \Omega\right)>0$.

To estimate the $L^{m}\left(\Gamma_{1}\right)$ norm of $u$ we introduce the Sobolev space of fractional order $H^{s}(\Omega)$, where $s>0$ is a free parameter, to be chosen later. We recall the embedding (see [1, Theorem 5.8])

$$
\|u\|_{l, \Gamma_{1}} \leq C_{3}\|u\|_{H^{s}(\Omega)}
$$

with $C_{3}=C_{3}(l, s, \Omega)>0$, which holds for $l \geq 1$ and

$$
s=\frac{n}{2}-\frac{n-1}{l}>0 .
$$


Then, using the Hölder inequality on $\Gamma_{1}$,

$$
\|u\|_{m, \Gamma_{1}} \leq C_{4}\|u\|_{H^{s}(\Omega)},
$$

$C_{4}=C_{4}(m, s, \Omega)>0$, for

$$
0<s<1, \quad s \geq \frac{n}{2}-\frac{n-1}{m} .
$$

Next, by the interpolation (see [27, p. 49]) and Poincaré inequalities (see [37])

$$
\|u\|_{H^{s}(\Omega)} \leq C_{5}\|u\|_{2}^{1-s}\|\nabla u\|_{2}^{s},
$$

$C_{5}=C_{5}(s, \Omega)>0$. Then

$$
\|u\|_{m, \Gamma_{1}} \leq C_{6}\|u\|_{2}^{1-s}\|\nabla u\|_{2}^{s}
$$

for some $C_{6}=C_{6}(m, s, \Omega)>0$. By (48) and (50), using the Hölder inequality and the fact that $p>2$,

$$
I_{3} \leq C_{7}\left(\left\|u_{t}\right\|_{\mu, \Gamma_{1}, \alpha}^{\mu-1}+\left\|u_{t}\right\|_{m, \Gamma_{1}, \alpha}^{m-1}\right)\|u\|_{p}^{1-s}\|\nabla u\|_{2}^{s},
$$

with $C_{7}=C_{7}\left(\mu, m, p, s, c_{1},\|\alpha\|_{\infty}, \Omega\right)>0$. By Young's inequality, if

$$
s<\frac{2}{m}
$$

then, for any $\delta>0$,

$$
\begin{aligned}
I_{3} & \leq C_{7}\left(C(\delta)\left\|u_{t}\right\|_{\mu, \Gamma_{1}, \alpha}^{\mu}+\delta\|\nabla u\|_{2}^{2}+\delta\|u\|_{p}^{p}\right)\|u\|_{p}^{1-s-p(1 / \mu-s / 2)} \\
& +C_{7}\left(C(\delta)\left\|u_{t}\right\|_{m, \Gamma_{1}, \alpha}^{m}+\delta\|\nabla u\|_{2}^{2}+\delta\|u\|_{p}^{p}\right)\|u\|_{p}^{1-s-p(1 / m-s / 2)}
\end{aligned}
$$

for some $C(\delta)=C(\delta, \mu, m)>0$. By (45)-(46) we have $\|u\|_{p} \geq\left(\mathcal{H}_{0} p / c_{3}\right)^{1 / p}$, hence from $1-s-p\left(\frac{1}{\mu}-\frac{s}{2}\right) \leq 1-s-p\left(\frac{1}{m}-\frac{s}{2}\right)$,

$$
I_{3} \leq C_{8}\left[C(\delta)\left(\left\|u_{t}\right\|_{\mu, \Gamma_{1}, \alpha}^{\mu}+\left\|u_{t}\right\|_{m, \Gamma_{1}, \alpha}^{m}\right)+\delta\|\nabla u\|_{2}^{2}+\delta\|u\|_{p}^{p}\right]\|u\|_{p}^{1-s-p(1 / m-s / 2)},
$$

with $C_{8}=C_{8}\left(\mu, m, p, s, c_{1}, c_{3},\|\alpha\|_{\infty}, \Omega, \mathcal{H}_{0}\right)>0$. Next, if $1-s-p\left(\frac{1}{m}-\frac{s}{2}\right)<0$, that is if

$$
s<\left(\frac{p}{m}-1\right) /\left(\frac{p}{2}-1\right)
$$

using (46) and setting $\overline{\alpha_{s}}=-\left[1-s-p\left(\frac{1}{m}-\frac{s}{2}\right)\right] / p>0$, we obtain

$$
I_{3} \leq C_{8}\left[C(\delta)\left(\left\|u_{t}\right\|_{\mu, \Gamma_{1}, \alpha}^{\mu}+\left\|u_{t}\right\|_{m, \Gamma_{1}, \alpha}^{m}\right)+\delta\|\nabla u\|_{2}^{2}+\delta\|u\|_{p}^{p}\right] \mathcal{H}^{-\overline{\alpha_{s}}}
$$


(with a possibly different value of $C_{8}$ ). Then, by (Q3) and (45), for $0<\alpha_{s}<\overline{\alpha_{s}}$,

$$
\begin{aligned}
I_{3} & \leq C_{8}\left[c_{4} C(\delta) \mathcal{H}^{\prime}+\delta\|\nabla u\|_{2}^{2}+\delta\|u\|_{p}^{p}\right] \mathcal{H}^{-\bar{\alpha}_{s}} \\
& \leq C_{9}\left[C(\delta) \mathcal{H}^{\prime} \mathcal{H}^{-\alpha_{s}}+\delta\|\nabla u\|_{2}^{2}+\delta\|u\|_{p}^{p}\right]
\end{aligned}
$$

where $C_{9}=C_{9}\left(\mu, m, p, s, \alpha_{s}, c_{1}, c_{3}, c_{4},\|\alpha\|_{\infty}, \Omega, \mathcal{H}_{0}\right)>0$. Choosing $\delta \leq \min \left\{c_{7} / 2 C_{9}\right.$, $\left.c_{6} / 2 C_{9}\right\}$, this estimate, combined with (47), gives

$$
I_{2} \geq 2\left\|u_{t}\right\|_{2}^{2}+\frac{c_{6}}{2}\|u\|_{p}^{p}+\frac{c_{7}}{2}\|\nabla u\|_{2}^{2}+2 \mathcal{H}-C_{10} \mathcal{H}^{\prime} \mathcal{H}^{-\alpha_{s}}
$$

where $C_{10}=C_{9} C(\delta)$. From this point the proof can be completed as in [11] or [25], provided we can show the existence of a value of the parameter $s$ satisfying (49), (51) and (52). When $1<m \leq 2$ it is enough to verify that $\frac{n}{2}-\frac{n-1}{m} \leq 1$, which is clearly true. When $m>2$, since

$$
\left(\frac{p}{m}-1\right) /\left(\frac{p}{2}-1\right) \leq \frac{2}{m} \leq 1
$$

it is enough to verify that $m<p$ and

$$
\frac{n}{2}-\frac{n-1}{m}<\left(\frac{p}{m}-1\right) /\left(\frac{p}{2}-1\right)
$$

which is exactly (11). An easy calculation shows that $m_{0}(p)<p$ for $p>2$, concluding the proof.

\section{REFERENCES}

1. R. A. Adams, Sobolev spaces (Academic Press, New York, 1975).

2. J. M. Ball, Finite time blow-up in nonlinear problems Nonlinear evolution equations, Proc. Symp., Madison/Wis., 1978, 189-205.

3. V. Barbu, Nonlinear semigroups and differential equations in Banach spaces (Noordhoff, Amsterdam, 1976).

4. H. Brézis, Analyse fonctionelle, théorie et applications (Masson, Paris, 1983).

5. G. Chen, Control and stabilization for the wave equation in a bounded domain, SIAM J. Control Optim. 17 (1979), 66-81.

6. G. Chen, Energy decay estimates and exact bounday value controllability for the wave equation in a bounded domain, J. Math. Pures Appl. 58 (1979), 249-273.

7. G. Chen, Control and stabilization for the wave equation in a bounded domain, part II, SIAM J. Control Optim. 19 (1981), 114-122.

8. G. Chen, A note on the boundary stabilization of the wave equation, SIAM J. Control Optim. 19 (1981), no. 1, 106-113.

9. T. Levi Civita and U. Amaldi, Lezioni di meccanica razionale, Vol. 2 (Zanichelli Editore, Bologna, 1929), second ed.

10. R. E. Edwards, Functional analysis, theory and applications (Holt, Rinehart and Winston, New York, 1965).

11. V. Georgiev and G. Todorova, Existence of a solution of the wave equation with nonlinear damping and source terms, J. Differential Equations 109 (1994), 295-308. 
12. R. T. Glassey, Blow-up theorems for nonlinear wave equations, Math. Z. 132 (1973), 183-203.

13. K. Jorgens, Nonlinear wave equations (Lectures Notes, University of Colorado, 1970).

14. J. B. Keller, On solutions of nonlinear wave equations, Comm. Pure Appl. Math. 10 (1957), 523-530.

15. R. J. Knops, H. A. Levine, and L. E. Payne, Nonexistence, instability, and growth theorems for solutions of a class of abstract nonlinear equations with applications to nonlinear elastodynamic, Arch. Rational Mech. Anal. 55 (1974), no. 1, 52-72.

16. V. Komorkin and E. Zuazua, A direct method for boundary stabilization of the wave equation, J. Math. Pures Appl. 69 (1990), 33-54.

17. J. Lagnese, Decay of solutions of wave equations in a bounded region with boundary dissipation, J. Differential Equations 50 (1983), 163-182.

18. J. Lagnese, Note on boundary stabilization of wave equations, SIAM J. Control Optim. 26 (1988), 1250-1256.

19. I. Lasiecka, Stabilization of hyperbolic and parabolic systems with nonlinearly perturbed boundary condition, J. Differential Equations 75 (1988), 53-87.

20. I. Lasiecka and D. Tataru, Uniform boundary stabilization of semilinear wave equations with nonlinear boundary damping, Differential Integral Equation 6 (1993), no. 3, $507-533$.

21. H. Levine, Nonexistence of global solutions of nonlinear wave equations Improperly posed boundary value problems (Pitman, 1975), 94-104.

22. H. A. Levine, Some additional remarks on the nonexistence of global solutions to nonlinear wave equations, SIAM J. Math. Anal. 5 (1974), 138-146.

23. H. A. Levine and L. E. Payne, Nonexistence theorems for the heat equation with nonlinear boundary conditions and for the porous medium equation backward in time, $J$. Differential Equations 16 (1974), 319-334.

24. H. A. Levine and L. E. Payne, Some nonexistence theorems for initial-boundary value problems with nonlinear boundary constraints, Proc. Amer. Math. Soc. 46 (1974), 277284.

25. H. A. Levine and J. Serrin, Global nonexistence theorems for quasilinear evolution equations with dissipation, Arch. Rational Mech. Anal. 137 (1997), 341-361.

26. J. L. Lions, Quelques méthodes de résolutions des problémes aux limites non linéaires (Dunod, Paris, 1969).

27. J. L. Lions and E. Magenes, Problémes aux limites non homogénes, Vol. 1 (Dunod, Paris, 1968).

28. R. H. Martin, Nonlinear operators and differential equations in Banach spaces (Wiley and Sons, New York, 1976).

29. L. E. Payne and D. H. Sattinger, Saddle points and instability of nonlinear hyperbolic equations, Israel J. Math. 22 (1975), 273-303.

30. P. Pucci and J. Serrin, Global nonexistence for abstract evolution equations with positive initial energy, J. Differential Equations 150 (1998), no. 1, 203-214.

31. H. L. Royden, Real analysis (MacMillan, New York, 1963).

32. I. Segal, Nonlinear semigroups, Ann. of Math. 78 (1963), 339-364.

33. M. Struwe, Variational methods (Springer-Verlag, 1990).

34. M. Tsutsumi, On solutions of semilinear differential equations in a Hilbert space, Math. Japon. 17 (1972), 173-193.

35. E. Vitillaro, Global nonexistence theorems for a class of evolution equations with dissipation and application, Arch. Rational Mech. Anal. 149 (1999), 155-182.

36. E. Vitillaro, Some new results on global nonexistence and blow-up for evolution problems with positive initial energy, Rend. Ist. Mat. Univ. Trieste 31 Suppl. 2 (2000), 245275.

37. W. P. Ziemer, Weakly differentiable functions, Graduate Text in Mathematics, Vol. 120 (Springer-Verlag, 1989).

38. E. Zuazua, Uniform stabilization of the wave equation by nonlinear boundary feedback, SIAM J. Control Optim. 28 (1990), 466-477. 